\title{
RINGS ON WATER AND THEIR ENTROPY
}

\author{
MICHEL MENDÈS FRANCE ${ }^{\bowtie}$ and TADASHI TOKIEDA
}

(Received 1 January 2011; accepted 16 December 2011; first published online 14 March 2013)

Communicated by I. E. Shparlinski

A la mémoire d'Alf van der Poorten

\begin{abstract}
We introduce the entropy of a family of planar curves in terms of the number of intersections of the family with a random line, calculate it for key examples, and discuss the entropy of a pattern of rings produced by an impulse on the surface of still water.
\end{abstract}

2010 Mathematics subject classification: primary 60D05; secondary 76B10.

Keywords and phrases: entropy, geometric probability, water wave, sound, raindrop.

\section{Disturbance on water}

A raindrop or a falling stone-how complex are the disturbances they excite on the surface of still water? We quantify disturbance by proposing a definition of entropy of a family of planar curves in Section 2, which is normalized to handle diverging or converging concentric rings in Section 3. The spread rates of rings generated by an impulse on water are determined by a simple estimate in Section 4. These results are combined in Section 5 to conclude that the disturbance on water has an entropy independent of the wave type, and that this entropy is in fact maximal. On the other hand, the sound emitted by the impulse does depend on whether the source was a raindrop or a falling stone, and Section 6 gives an order-of-magnitude analysis of different pitches.

A haiku of Bashou from 1686 (see Figure 1) touched on this circle of problems.

\section{Entropy of planar curves}

Let $\Gamma=\left\{\gamma_{1}, \gamma_{2}, \ldots\right\}$ be a family of rectifiable curves in the plane such that the sum of the lengths $|\Gamma|=\sum_{n}\left|\gamma_{n}\right|$ converges. We denote the convex hull of $\bigcup_{n} \gamma_{n}$ by $C(\Gamma)$ and the boundary of $C(\Gamma)$ by $\partial C(\Gamma)$.

(c) 2013 Australian Mathematical Publishing Association Inc. 1446-7887/2013 \$16.00 


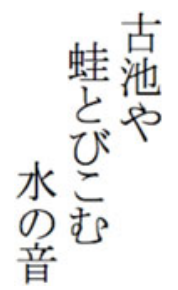

FIGURE 1. A haiku of Bashou.

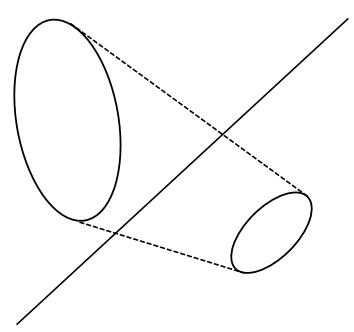

Figure 2. Permeable $\Gamma: L \cap C(\Gamma) \neq \emptyset$ yet $L \cap \Gamma=\emptyset$.

A line $L$ in the plane is specified by its distance $r$ to the origin and the angle $\theta$ its normal makes with the horizontal. The space of lines that intersect $C(\Gamma)$ is endowed with a (conditional) probability measure $\left(\int_{L: L \cap C(\Gamma) \neq \emptyset} d r d \theta\right)^{-1} d r d \theta$. Write $p_{n}$ for the probability that a random line $L$ intersects $\Gamma$ in exactly $n$ points. Then the Shannon entropy $h(\Gamma)$ of $\Gamma$ is

$$
h(\Gamma)=-\sum_{n \geqslant 0} p_{n} \log p_{n},
$$

with the usual convention that $0 \times \log 0=0$. Higher-dimensional generalizations suggest themselves, but we shall stick to planar curves.

First, we bound the entropy. We say that $\Gamma$ is impermeable when $L \cap C(\Gamma)=\emptyset$ if and only if $L \cap \Gamma=\emptyset$. We give an example of a permeable $\Gamma$ in Figure 2.

THEOREM 1. The Shannon entropy is bounded above:

$$
h(\Gamma) \leqslant \log \left(\frac{2|\Gamma|}{|\partial C(\Gamma)|}+1\right)+1 .
$$

Moreover, if $\Gamma$ is impermeable, then the bound can be sharpened:

$$
h(\Gamma) \leqslant \log \frac{2|\Gamma|}{|\partial C(\Gamma)|}+1 .
$$

Remark 2. Via an extension of a classical theorem of Steinhaus [10], we recognize the quotient inside the logarithm as the expected number of intersections of $\Gamma$ with a random line: $2|\Gamma| /|\partial C(\Gamma)|=\sum_{n \geqslant 0} n p_{n}$. 
Proof. Using Lagrange multipliers to maximize the function $-\sum_{n \geqslant 0} x_{n} \log x_{n}$, subject to the constraints $x_{n} \geqslant 0, \sum_{n \geqslant 0} x_{n}=1$, and $\sum_{n \geqslant 0} n x_{n}=E$, we find that the maximum is attained when $x_{n}=(1+1 / E)^{-n-1} / E$. If $\Gamma$ is impermeable, then $p_{0}=0$ and the maximum is attained when $x_{n}=(1-1 / E)^{n-1} / E$.

We define the entropy $H(\Gamma)$ of $\Gamma$ by

$$
H(\Gamma):=\frac{h(\Gamma)}{\log (2|\Gamma| /|\partial C(\Gamma)|+1)+1}
$$

or, if $\Gamma$ is impermeable, by

$$
H(\Gamma):=\frac{h(\Gamma)}{\log (2|\Gamma| /|\partial C(\Gamma)|)+1} .
$$

The bounds above imply that $0 \leqslant H(\Gamma) \leqslant 1$. Thus our entropy quantifies complexity more compactly than its thermodynamic namesake, whose range is unbounded above. In the image of rings on water, low entropy (that is, $H \approx 0$ ) should correspond to simple disturbance, and high entropy (that is, $H \approx 1$ ) to complex disturbance. Note that, in contrast to entropy, energy quantifies the size rather than the complexity of disturbance. By construction, $H$ is invariant under rescaling (homothety) of the plane.

\section{Families of concentric rings}

Suppose that $\Gamma=\left\{\gamma_{1}, \gamma_{2}, \ldots\right\}$ is a family of concentric circles (rings) of radii $r_{1}$, $r_{2}, \ldots$, and $\Gamma_{M}^{N}$, where $M<N$, is the subfamily $\left\{\gamma_{M}, \gamma_{M+1}, \ldots, \gamma_{N}\right\}$ of $\Gamma$. We derive formulae for the Shannon entropy of the subfamily $\Gamma_{M}^{N}$ in two cases: when $r_{n}$ increases to $\infty$ (diverging rings), and when $r_{n}$ decreases to 0 (converging rings). We then give a normalized definition for the entropy of $\Gamma$ itself.

Case 1: $r_{n} \rightarrow \infty$. The probability that a random line intersects $\Gamma_{M}^{N}$ in exactly $2 n$ points, where $1 \leqslant n \leqslant N-M$, is

$$
p_{2 n}=\frac{r_{N-n+1}-r_{N-n}}{r_{N}}
$$

setting $r_{M}=0$ for convenience so that $\sum_{n=1}^{N-M} p_{2 n}=1$. The Shannon entropy of $\Gamma_{M}^{N}$ is

$$
\begin{aligned}
h\left(\Gamma_{M}^{N}\right) & =-\sum_{n=1}^{N-M} \frac{r_{N-n+1}-r_{N-n}}{r_{N}} \log \frac{r_{N-n+1}-r_{N-n}}{r_{N}} \\
& =-\sum_{n=M}^{N-1} \frac{r_{n+1}-r_{n}}{r_{N}} \log \frac{r_{n+1}-r_{n}}{r_{N}} \\
& =\log r_{N}-\frac{1}{r_{N}} \sum_{n=M}^{N-1}\left(r_{n+1}-r_{n}\right) \log \left(r_{n+1}-r_{n}\right) .
\end{aligned}
$$


Case 2: $r_{n} \rightarrow 0$. This time, setting $r_{N}=0$,

$$
p_{2 n}=\frac{r_{M+n-1}-r_{M+n}}{r_{M}}
$$

when $1 \leqslant n \leqslant N-M$. Hence

$$
h\left(\Gamma_{M}^{N}\right)=\log r_{M}-\frac{1}{r_{M}} \sum_{n=M}^{N-1}\left(r_{n}-r_{n+1}\right) \log \left(r_{n}-r_{n+1}\right) .
$$

In these cases, unlike in Section 2, $h(\Gamma)$ may become infinite. To obtain a finite value, it is natural to normalize the definition of entropy as follows:

$$
H(\Gamma):=\lim _{\lambda \rightarrow \infty} \lim _{M \rightarrow \infty} \frac{h\left(\Gamma_{M}^{\lambda M}\right)}{\log \left(2\left|\Gamma_{M}^{\lambda M}\right| / \max _{n \in[M, \lambda M]}\left|\gamma_{n}\right|\right)+1}
$$

When the limits fail to exist, upper and lower entropies are defined using lim sup and lim inf.

REMARK 3. First, in earlier papers [3-7], the first-named author chose a slightly different definition of entropy: $h\left(\Gamma_{M}^{\lambda M}\right)$ was normalized by the factor $\log \left|\Gamma_{M}^{\lambda M}\right|$ rather than by $\log \left(2\left|\Gamma_{M}^{\lambda M}\right| / \max _{n}\left|\gamma_{n}\right|\right)+1$. In the information-theoretic context, a list of canonically desirable properties forces a unique definition of entropy. Here, we are attempting to model a concept of complexity in a new context where desirable properties are less canonical, so there is freedom in the definitions adopted. Exitus acta probat.

Second, we comment on the subfamily $\Gamma_{M}^{\lambda M}$. Take the case when $r_{n} \rightarrow \infty$. Intuitively $M, N \rightarrow \infty$ in $\Gamma_{M}^{N}$ means that we are zooming out as the wave expands and bringing more and more rings into our view. This motivates making $M, N$ grow in a coordinated fashion, as suitable functions of each other. The choice $N=\lambda M$ seems most natural, and leads to reasonable results. The case when $r_{n} \rightarrow 0$ is similar.

\section{Types of wave and spread rates}

An impulse applied to the surface of water excites a wave whose crests propagate as concentric rings. Their analysis was set as a prize problem by the Académie des Sciences de Paris in 1816 and treated by Cauchy and Poisson [1, 9].

Suppose that we are given some dispersion relation between the wave frequency $\omega$ and the wave number $k$. If the impulse is localized at the origin, then after time $t$ the water surface can be shown to have the profile

$$
z=\operatorname{Re} \frac{1}{2 \pi} \int_{0}^{\infty} \exp (i \omega(k) t) J_{0}(k r) k d k
$$

in cylindrical coordinates $r, \theta, z$ (the variable $\theta$ is ignorable). Kelvin [12] pioneered the method of stationary phase to approximate such integrals (Stokes did some even 

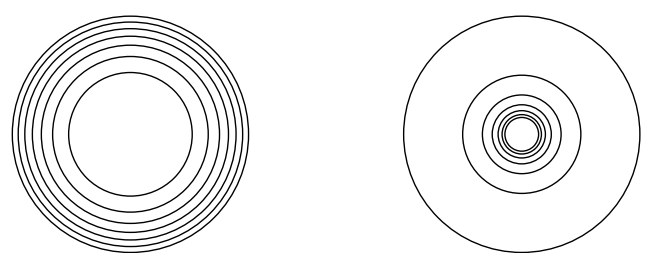

FigURE 3 . Rings of capillary wave and gravity wave $(1 \leqslant n \leqslant 7)$.

earlier work [11]). But here we can resort to a short-cut, because we are interested only in spacing between crests and not in amplitude: all we need is the condition that $k r_{n}-\omega t \in 2 \pi \mathbb{Z}$ for the phase of the $n$th crest, extracting the representative $k$ from the group velocity equation

$$
\frac{r_{n}}{t}=\frac{d \omega}{d k}
$$

The approximation is accurate provided $\omega$ in the neighborhood of this representative $k$ is close to being quadratic, that is, provided the dimensionless test ratio is small:

$$
\frac{d^{3} \omega}{d k^{3}} / \sqrt{t\left|\frac{d^{2} \omega}{d k^{2}}\right|^{3}} \ll 1 .
$$

When $\omega \propto k^{a}$ (where $a \neq 1$ ), we find that

$$
\frac{r_{n}}{t} \propto k^{a-1}, \quad 2 \pi n=\left|k r_{n}-\omega t\right| \propto\left(\frac{r_{n}}{t}\right)^{1 /(a-1)} r_{n}, \quad r_{n}=C n^{1-1 / a},
$$

where $C$ denotes a constant that may not be the same at each occurrence.

For a capillary wave (a raindrop), the relevant parameters being the surface tension $\sigma$ and the density $\rho$ of water, we can see dimensionally that the dispersion relation must be of the form $\omega \sim \sqrt{\sigma k^{3} / \rho}$. The power $a$ is $3 / 2$, so

$$
r_{n} \sim\left(\frac{27 \sigma t^{2} \pi n}{2 \rho}\right)^{1 / 3}=C n^{1 / 3}
$$

accurately provided that $\sqrt{\sigma t^{2} / \rho r^{3}} \ll 1$. The radius $r_{n}$ is increasing in $n$, which means that for a capillary wave, $r_{1}, r_{2}, \ldots$ are ordered from the innermost ring, gradually crowding outward (Figure 3). For a gravity wave (a falling stone), $\omega \sim \sqrt{g k}$, where $g$ is the gravitational acceleration. The power $a$ is $1 / 2$, so

$$
r_{n} \sim \frac{g t^{2}}{8 \pi n}=C n^{-1}
$$

accurately provided that $\sqrt{2 r / g t^{2}} \ll 1$. The radius $r_{n}$ is decreasing in $n$, which means that for a gravity wave, $r_{1}, r_{2}, \ldots$ are ordered from the outermost ring, gradually crowding inward (Figure 3 ). 
To get the dispersion relations between $\omega$ and $k$ above, we got away with dimensional analysis. These relations are in fact the deep-water limits, $k h \gg 1$, of the exact relation

$$
\omega^{2}=\left(\sigma k^{3} / \rho+g k\right) \tanh (k h),
$$

where $h$ is the depth of the water. Our approximate estimates are endorsed by a full analysis by the method of Kelvin using this exact dispersion relation.

\section{5. $H$ for polynomial and exponential families}

Recall from Section 3 that $N=\lambda M$. Throughout this section, $\alpha>0$.

ExAmple 4. Suppose that $r_{n}=n^{\alpha}$. Then

$$
\begin{aligned}
h\left(\Gamma_{M}^{N}\right) & =\log N^{\alpha}-\frac{1}{N^{\alpha}} \sum_{n=M}^{N-1} \alpha n^{\alpha-1} \log \alpha n^{\alpha-1}+o(\log N) \\
& =\alpha \log N-\frac{\alpha(\alpha-1)}{N^{\alpha}} \int_{M}^{N-1} x^{\alpha-1} \log x d x+o(\log N) \\
& =\alpha \log N-\frac{\alpha-1}{N^{\alpha}}\left(N^{\alpha} \log N-M^{\alpha} \log M\right)+o(\log N) \\
& =\log N+(\alpha-1)\left(\frac{M}{N}\right)^{\alpha} \log M+o(\log N) .
\end{aligned}
$$

We introduce a normalizing factor of $\log \left(2 \sum_{n=M}^{N} n^{\alpha} / N^{\alpha}\right)+1$, which can be seen to be equal to $\log N+\log \left(1-(M / N)^{\alpha+1}\right)+o(\log N)$, and it follows that

$$
H(\Gamma)=\lim _{\lambda \rightarrow \infty} \lim _{M \rightarrow \infty} \frac{\log \lambda+\left(1+\frac{\alpha-1}{\lambda^{\alpha}}\right) \log M}{\log \lambda+\log M+\log \left(1-\frac{1}{\lambda^{\alpha+1}}\right)}=\lim _{\lambda \rightarrow \infty}\left(1+\frac{\alpha-1}{\lambda^{\alpha}}\right)=1 .
$$

Example 5. Suppose that $r_{n}=n^{-\alpha}$. Mimicking the steps of Example 4,

$$
h\left(\Gamma_{M}^{\lambda M}\right)=\log M-\frac{\alpha+1}{\lambda^{\alpha}} \log (\lambda M)+o(\log M) .
$$

If $\alpha \neq 1$, then

$$
\sum_{n=M}^{\lambda M} n^{-\alpha} / M^{-\alpha}=M\left(\lambda^{1-\alpha}-1\right) /(1-\alpha)+o(M)
$$

so $H(\Gamma)=1$. If $\alpha=1$, then

$$
\sum_{n=M}^{\lambda M} n^{-1} / M^{-1}=M \log \lambda+o(M)
$$

so again $H(\Gamma)=1$. 


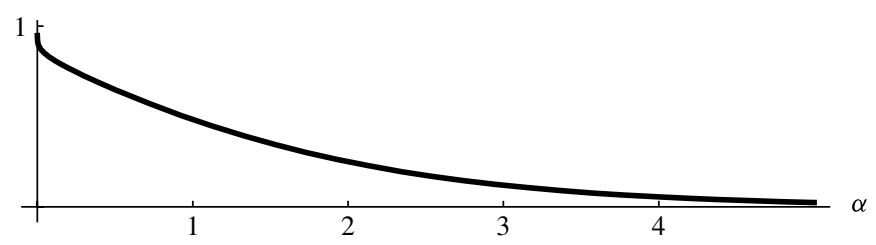

FIGURE 4. Entropies for exponential families.

Together with the estimates on capillary and gravity waves from Section 4, we see that both a raindrop (Example 4, where $\alpha=1 / 3$ ) and a falling stone (Example 5, where $-\alpha=-1)$ excite disturbances of the same maximal entropy 1 on the surface of still water.

Of course, there exist families for which $H(\Gamma)<1$. To wit, consider exponentially diverging rings.

ExAmple 6. Suppose that $r_{n}=e^{\alpha n}$. Then

$$
h\left(\Gamma_{M}^{N}\right)=\alpha N-\alpha\left(e^{\alpha}-1\right) e^{-\alpha N} \sum_{n=M}^{N-1} n e^{\alpha n}-\left(e^{\alpha}-1\right) \log \left(e^{\alpha}-1\right) e^{-\alpha N} \sum_{n=M}^{N-1} e^{\alpha n} .
$$

The latter geometric sum is equal to $\left(e^{\alpha N}-e^{\alpha M}\right) /\left(e^{\alpha}-1\right)$, while the former is equal to

$$
\frac{\partial}{\partial \alpha} \frac{e^{\alpha N}-e^{\alpha M}}{e^{\alpha}-1}=\frac{N e^{\alpha N}-M e^{\alpha M}}{e^{\alpha}-1}-\left(e^{\alpha N}-e^{\alpha M}\right) \frac{e^{\alpha}}{\left(e^{\alpha}-1\right)^{2}} .
$$

Hence

$$
h\left(\Gamma_{M}^{\lambda M}\right)=\alpha M e^{-\alpha(\lambda-1) M}+\left(\frac{\alpha e^{\alpha}}{e^{\alpha}-1}-\log \left(e^{\alpha}-1\right)\right)\left(1-e^{-\alpha(\lambda-1) M}\right),
$$

and, on normalizing by $\log \left(2 \sum_{n=M}^{\lambda M} e^{\alpha n} / e^{\alpha \lambda M}\right)+1$, it follows that

$$
H(\Gamma)=\frac{\alpha\left(e^{\alpha}-1\right)^{-1}-\log \left(1-e^{-\alpha}\right)}{1+\log 2-\log \left(1-e^{-\alpha}\right)} .
$$

As the plot of $H$ against $\alpha$ shows (Figure 4), every value between 0 and 1 is realized as the entropy of some exponential family. Speciously, the entropy of an exponential family converges to the common entropy 1 of polynomial families as $\alpha$ is reduced to 0 , though the vertical tangency when $\alpha=0$ bears witness to the gap that distinguishes these spread rates.

ExAmple 7. Suppose that $r_{n}=e^{-\alpha n}$. For exponentially converging rings, rescaling invariance tells us that $H$ is identical in form to the result of Example 6. (Rescaling is inapplicable between Examples 4 and 5.) 

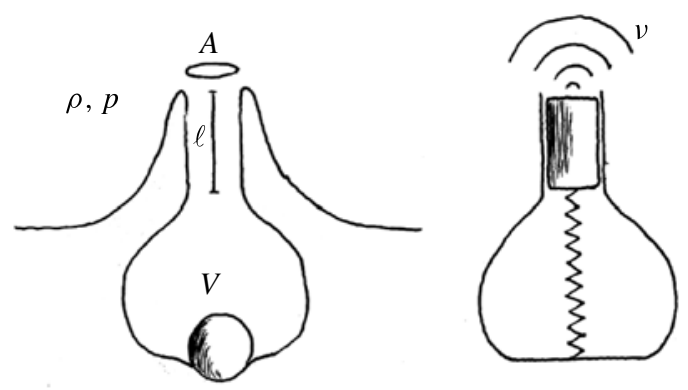

Figure 5. Acoustics in a cavity, and a spring in a bottle.

Example 8. Suppose that $r_{n}=(\log n)^{ \pm \alpha}$. Such rings turn out to have $H=0$, and $H\left(r_{n}=1\right)=0$ trivially from the definition of the Shannon entropy.

We end this section with a question: given an arbitrary value between 0 and 1 , can we drive a point on the surface of water according to some function of time $t$ so that the entropy of the generated rings takes that value? For example, we have seen that $H=1$ can be generated by $\delta(t)$. We believe the answer is yes.

\section{Acoustic appendix}

A body of diameter $d$ falling on water emits a sound- 'splitch' for a raindrop, 'glop' for a stone. How does the pitch of the sound depend on $d$ ? On impact the body dents the water, forming a cavity that is quickly pinched to a bubble. The body's wettability influences the type of splash produced [2]; for the estimate below to work, it is necessary only that a cavity be formed as in Figure 5, which happens whenever the contact angle of the water against the body is neither too large nor too small. What is responsible for the sound is the oscillation of air inside the cavity. Let us try a naive model that the air in the bulb of the cavity, of volume $V$, acts like a spring, while the air in the neck of the cavity, of length $l$ and cross-sectional area $A$, acts like a mass attached to this spring. Write $\rho$ and $p$ for the density and pressure of air, $\Delta p$ for the pressure difference between outside and inside the cavity, and say the air in the neck is displaced vertically by $z$. The air in the bulb has compressibility $\kappa$, given by

$$
\kappa=-\frac{\partial V / \partial p}{V} \approx-\frac{A z / \Delta p}{V}
$$

But $\kappa=1 / \gamma p$ for a perfect gas (where $\gamma$ denotes the ratio of specific heats). So we can read off the frequency $v$ from the spring equation $\rho l A \ddot{z}=A \Delta p$, namely,

$$
2 \pi v \approx \sqrt{\frac{A}{V l} \frac{\gamma p}{\rho}} .
$$


Now $\sqrt{\gamma p / \rho}$ is the speed of sound in air, which is $345 \mathrm{~m} / \mathrm{s}$ at $22^{\circ} \mathrm{C}$, comfortably warm for Bashou's frog. Substituting our cavity geometry, $l \approx d, A \approx d^{2}, V \approx(2 d)^{3}$, we get

$$
v \approx \frac{345 \times 100}{2 \pi \sqrt{8} d}=C d^{-1},
$$

where $d$ is measured in centimeters. This predicts $647 \mathrm{~Hz}$ or $\mathrm{E}_{5}$ ('middle C' is $\mathrm{C}_{4}$ ) for a stone of diameter $3 \mathrm{~cm}$, and $6.47 \mathrm{kHz}$ for a raindrop of diameter $0.3 \mathrm{~cm}$. The accord with observation is surprisingly fair: after all, a mere factor of 2 would have shifted the estimated note by a whole octave.

Blowing across the top of a bottle as a wind instrument involves similar acoustics; [8, Ch. XII, Section 6] contains an analysis based on energy rather than on force, essentially equivalent to ours. For a bottle of Bordeaux with $l \approx 8 \mathrm{~cm}, A \approx \pi \mathrm{cm}^{2}$, $V \approx 700 \mathrm{~cm}^{3}$, the predicted $v$ is $130 \mathrm{~Hz}$ or $\mathrm{C}_{3}$. Tuned to the Steinway of Trinity Hall, the observed pitch was $\mathrm{B}_{2}$ - just one note off.

\section{Acknowledgements}

We thank Joan Mendès France and Lisa Willis for their help regarding IT matters.

\section{References}

[1] A.-L. Cauchy, 'Mémoire sur la théorie de la propagation des ondes à la surface d'un fluide pesant d'une profondeur indéfinie', Mém. Acad. R. Sci. 1 (1827), 3-312.

[2] C. Duez, C. Ybert, C. Clanet and L. Bocquet, 'Making a splash with water repellency', Nat. Phys. 3 (2007), 180-183.

[3] M. Mendès France, 'Chaotic curves', in: Rhythms in Biology and Other Fields of Application, Lecture Notes in Biomath, 49 (Springer, Berlin, 1983), pp. 352-367.

[4] M. Mendès France, 'Entropie, dimension et thermodynamique des courbes planes', in: Séminaire de Théorie des Nombres, Paris 1981-82. (Séminaire Delange-Pisot-Poitou) (Birkhäuser, Basel, 1983), pp. 153-177.

[5] M. Mendès France, 'Folding paper and thermodynamics', Phys. Rep. 103 (1984), 161-172.

[6] M. Mendès France, 'Chaos implies confusion', in: Number Theory and Dynamical Systems, LMS Lecture Notes, 134 (eds. M. Dodson and J. Vickers) (Cambridge University Press, Cambridge, 1989), pp. 137-152.

[7] M. Mendès France, 'The Planck constant of a curve', in: Fractal Geometry and Analysis, (eds. J. Bélair and S. Dubuc) (Kluwer Academic Publishers, Dordrecht, 1991), pp. 325-366.

[8] A. R. Paterson, A First Course in Fluid Dynamics (Cambridge University Press, Cambridge, 1983).

[9] S. D. Poisson, 'Mémoire sur la théorie des ondes', Mém. Acad. R. Sci. 1 (1816), 70-186.

[10] L. Santaló, Integral Geometry and Geometric Probability, 2nd edn (Cambridge University Press, Cambridge, 2002).

[11] G. G. Stokes, 'On the numerical calculation of a class of definite integrals and infinite series', Trans. Camb. Phil. Soc. 9 (1850), 379-407.

[12] W. Thomson, 'On the waves produced by a single impulse in water of any depth, or in a dispersive medium', Proc. R. Soc. 42 (1887), 80-83. 
MICHEL MENDÈS FRANCE, A2X Mathématiques, UMR 5465, Université de Bordeaux 1, 33405 Talence Cedex, France

e-mail: michel.mendes-france@math.u-bordeaux1.fr

TADASHI TOKIEDA, Trinity Hall, Cambridge CB2 1TJ, UK e-mail: tokieda@dpmms.cam.ac.uk 